\title{
Radar adjusted data versus modelled precipitation: a case study over Cyprus
}

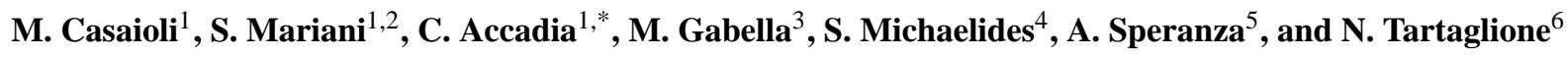 \\ ${ }^{1}$ Agenzia per la Protezione dell' Ambiente e per i Servizi Tecnici (APAT), Rome, Italy \\ ${ }^{2}$ Department of Mathematics, University of Ferrara, Ferrara, Italy \\ ${ }^{3}$ Department of Electronics, Politecnico di Torino, Turin, Italy \\ ${ }^{4}$ Meteorological Service, Nicosia, Cyprus \\ ${ }^{5}$ Department of Mathematics and Informatics, University of Camerino, Camerino, Italy \\ ${ }^{6}$ Department of Physics, University of Camerino, Camerino, Italy \\ * present organization: EUMETSAT, Darmstadt, Germany
}

Received: 30 October 2005 - Revised: 28 December 2005 - Accepted: 29 December 2005 - Published: 30 January 2006

\begin{abstract}
In the framework of the European VOLTAIRE project (Fifth Framework Programme), simulations of relatively heavy precipitation events, which occurred over the island of Cyprus, by means of numerical atmospheric models were performed. One of the aims of the project was indeed the comparison of modelled rainfall fields with multi-sensor observations. Thus, for the 5 March 2003 event, the 24-h accumulated precipitation BOlogna Limited Area Model (BOLAM) forecast was compared with the available observations reconstructed from ground-based radar data and estimated by rain gauge data.
\end{abstract}

Since radar data may be affected by errors depending on the distance from the radar, these data could be rangeadjusted by using other sensors. In this case, the Precipitation Radar aboard the Tropical Rainfall Measuring Mission (TRMM) satellite was used to adjust the ground-based radar data with a two-parameter scheme. Thus, in this work, two observational fields were employed: the rain gauge gridded analysis and the observational analysis obtained by merging the range-adjusted radar and rain gauge fields.

In order to verify the modelled precipitation, both nonparametric skill scores and the contiguous rain area (CRA) analysis were applied. Skill score results show some differences when using the two observational fields. CRA results are instead quite in agreement, showing that in general a $0.27^{\circ}$ eastward shift optimizes the forecast with respect to the two observational analyses. This result is also supported by a subjective inspection of the shifted forecast field, whose gross features agree with the analysis pattern more than the non-shifted forecast one.

However, some open questions, especially regarding the effect of other range adjustment techniques, remain open and need to be addressed in future works.

Correspondence to: M. Casaioli

(marco.casaioli@apat.it)

\section{Introduction}

Meteorological observations, derived from different instruments, can be compared with model outputs in order to verify the models themselves and quantify their skill since, focusing on the relationship between observations and forecasts, it is possible to underline the model's ability to forecast correctly a meteorological event. Forecast verification can also provide insight into the way atmospheric processes are modelled.

Precipitation is often the object of verification studies: point measurements over land are compared with modelled precipitation while over the sea it is not possible, usually, to perform the same kind of comparison. Nevertheless, satellite or ground-based remote sensing can help evaluating rainfall over the sea.

Performing this kind of operation is one of the purposes of the European VOLTAIRE project (Fifth Framework Programme; http://www.voltaireproject.org). After selecting several relevant weather events over the island of Cyprus, precipitation measured at rain gauges (hereafter $R G$ ) and retrieved by both the ground-based radar (hereafter GR) and the Precipitation Radar on board the Tropical Rainfall Measuring Mission (TRMM) was collected. These case studies were also modelled using the hydrostatic BOlogna Limited Area Model (BOLAM).

In this work, we focus on the relatively heavy precipitation event occurred on 5 March 2003. This was due to a cyclonic circulation slowly moving from the western to the eastern Mediterranean Sea (Tartaglione et al., 2005).

Since GR data can be prone to distance-dependent errors, some range adjustment may be helpful. In this study, TRMM precipitation radar data were used to adjust the GR data, employing a (physically easy to be interpreted) two-coefficient scheme. The forecast field was compared with both a RG 


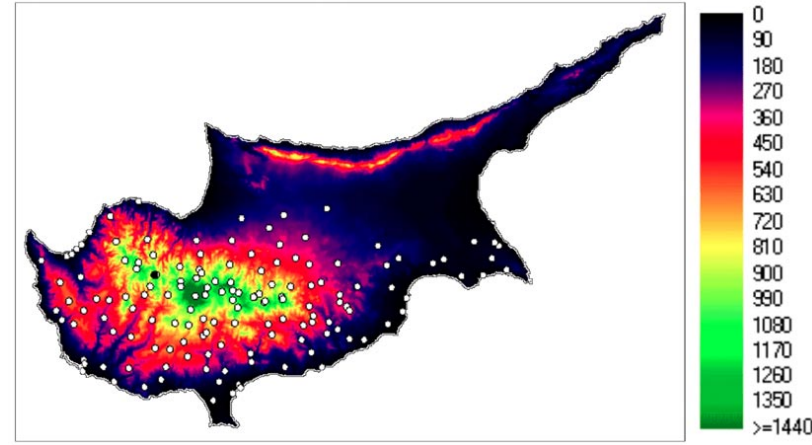

Fig. 1. Geographical distribution over the island of Cyprus of the 147 rain gauge stations, marked as white circles, and the location of the ground-based radar $\left(34.98^{\circ} \mathrm{N}, 32.73^{\circ} \mathrm{E}, 1310 \mathrm{~m}\right.$ above mean sea level), marked as a black circle. Orography is indicated in colour scale; visible in figure is the Troodos mountain range.

gridded analysis and an observed precipitation field obtained by merging the RG data with rainfall retrieved from the range-adjusted GR data.

Both non-parametric skill scores (Hanssen and Kuipers, 1965; Schaefer, 1990; Wilks, 1995; Stephenson, 2000) and the contiguous rain area analysis (CRA; Ebert and McBride, 2000) were applied for verifying the modelled precipitation.

The paper is organized as follows: in Sect. 2, the observational data set and the radar range adjustment scheme are discussed; the atmospheric model BOLAM is presented in Sect. 3; a brief discussion of the applied verification methodologies is proposed in Sect. 4; results and conclusions are, finally, reported in Sect. 5.

\section{Ground-based radar and rain gauge data}

In order to verify precipitation forecast by BOLAM over Cyprus, both the RG and GR data, which are managed by the Meteorological Service of Cyprus, were used. The RG network and the GR location are shown in Fig. 1.

For the selected event, precipitation recorded at the RG network during $24 \mathrm{~h}$, from 06:00 UTC 5 March to 06:00 UTC 6 March 2003, was considered. Since RG gives only point measurements, a two-pass Barnes scheme (Barnes, 1964, 1973; Koch et al., 1983) was used in order to obtain a gridded $0.09^{\circ}$ observational analysis: the first observational analysis to be compared with BOLAM was obtained by using only RG data (see Fig. 2). However, in this case, the use of only $\mathrm{RG}$ for forecast verification may be insufficient for at least two reasons.

First, RG are only available on Cyprus' western part and coastal grid points (where observed precipitation is spread by the Barnes procedure) may suffer an underestimation with respect to the internal grid points. Second, the precipitation comparison is limited by the presence of the surrounding sea.

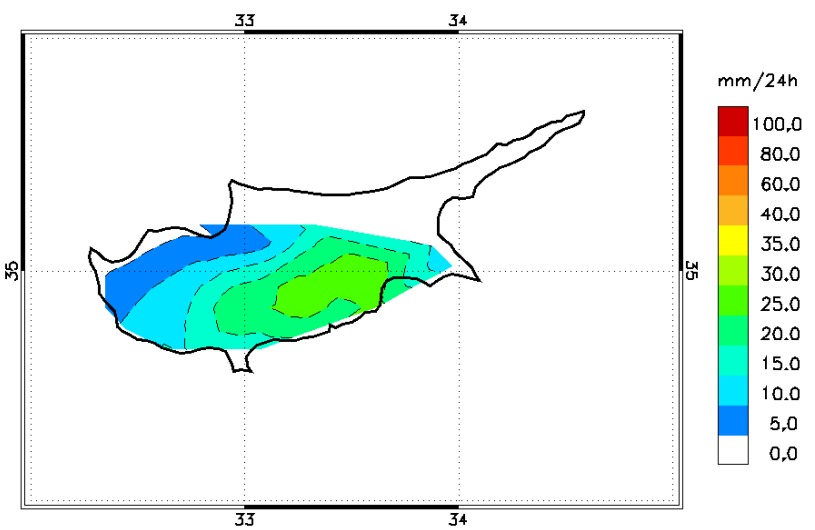

Fig. 2. The 24-h observational precipitation contours $(\mathrm{mm})$ from 06:00 UTC 5 March to 06:00 UTC 6 March 2003, obtained applying the Barnes analysis scheme.

The Cyprus GR can provide information also on precipitation over the waters surrounding the island of Cyprus. However, the proximity of the Troodos high massif in the southeastern part and of the Tripylos peak in the northwestern part (see Fig. 1) produced two obscured (no-data) radar sectors. Thus, in an attempt to obtain a better observational analysis over Cyprus and its surrounding sea, even considering that much precipitation occurred in the southeastern part of the island, we decided to merge GR data with the RG gridded analysis.

The GR raw data can be corrected and/or adjusted, since some factors can alter the beam response. GR measures rain from a lateral direction. In the present case, the distance between the echo and the radar varies from 10 to $120 \mathrm{~km}$. Because of this large ratio of distances, the scattering volume changes by a factor of over 100 , since the volume increases with the square of the distance. The scattering volume of TRMM has a similar size in all the locations. This advantage of TRMM suggests its use for estimating the influence of the GR sampling volume (Gabella et al., 2005). For both radars, the average reflectivity $Z$ in the same $10-\mathrm{km}$ circular ring is computed. Let us consider $<\mathrm{GR}>2 \pi$ and $<\mathrm{TRMM}>2 \pi$ the values of the average reflectivity, averaged in azimuth, for both GR and TRMM. Because of their size, these two variables show similar behaviour, except for the decreased sensitivity of GR with distance. Averaging over the large area of the rings reduces deviations caused by rain cells of high intensity.

Thus, the following factor:

$F=\frac{<\mathrm{GR}>_{2 \pi}}{<\mathrm{TRMM}>_{2 \pi}}$

is statistically described by using a weighted regression between $\log (F)$ and $\log (D)$, where $D$ is the distance between the echo and GR. The best relationship, among the ones 
found by Gabella et al. (2005), seems to be the following:

$$
\begin{aligned}
F_{d B} \equiv 10 \cdot \log (F) & =a_{0}+a_{1} \cdot \log \left(\frac{D}{D_{g}}\right)= \\
& =-4.1-10.1 \cdot \log \left(\frac{D}{40}\right),
\end{aligned}
$$

where $a_{0}$ and $a_{1}$ are the regression coefficients and $D_{g}$ is a coefficient for the normalization distance.

It is possible, this way, to use two different kinds of GR data: the original and the range-adjusted ones. The (original and adjusted) GR data were accumulated in the aforementioned time interval, then, in order to make them available on a latitude-longitude grid (with grid spacing of $0.09^{\circ}$ ); the remapping procedure (Baldwin, 2000; Accadia et al., 2003) was also applied.

However, in order to choose the best representative GR field, it was decided to compare the precipitation retrieved from the raw and the range-adjusted GR data with the rain gauge ones. Fig. 3 shows the results of this comparison. A near zero correlation is found when the original precipitation data is used in the comparison with the rain gauges (Fig. 3a), whereas a significantly (taking also into account its confidence interval) higher correlation is obtained by using precipitation reconstructed by range-adjusted GR data (Fig. 3b). In this latter case the better agreement between these two fields appears more evident. Therefore, we shall hereafter consider as GR data the range-adjusted ones.

Then, the observational analysis (OBSPREC; Fig. 4) is improved by merging the RG gridded field and the GR rangeadjusted data using the following formula for each verification grid point:

OBSPREC $=\frac{(\mathrm{GR} \times \mathrm{GR}+\mathrm{RG} \times \mathrm{RG})}{(\mathrm{GR}+\mathrm{RG})} ;$

this way, when there is no contribution from RG (over sea, for instance), OBSPREC is given only by GR; the same is true for RG, on the grid points where GR is shielded by mountains, for instance. When estimations by both instruments were available, they were respectively weighted by their values.

\section{Forecast precipitation}

The model used in this verification case study is BOLAM, a hydrostatic primitive-equation model (Malguzzi and Tartaglione, 1999; Buzzi and Foschini, 2000). The standard $6 \mathrm{~h}, 0.5^{\circ}$ resolution, 60-model-level ECMWF analyses were first horizontally interpolated onto the $30-\mathrm{km}$ model domain covering the entire Mediterranean region. The outputs of this model were used as initial and boundary conditions (one-way nesting) for a domain with a finer grid $(10 \mathrm{~km})$ and encompassing only the eastern Mediterranean region.

Modelled precipitation was then 24-h accumulated (see Fig. 5) for the same time interval described in the previous section. These data, originally on the BOLAM native grid, were remapped (Accadia et al., 2003) on the same grid of the

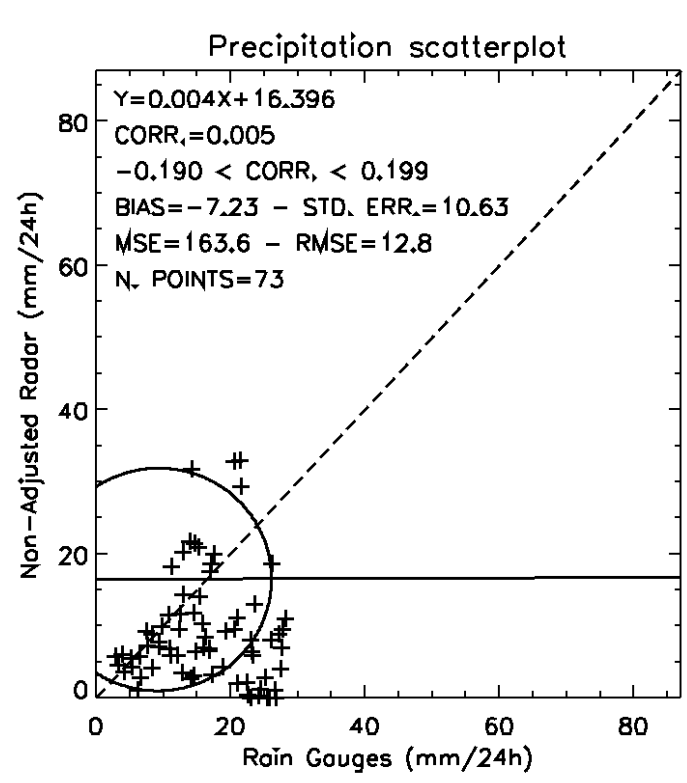

(a) RG gridded field vs. non-adjusted GR field

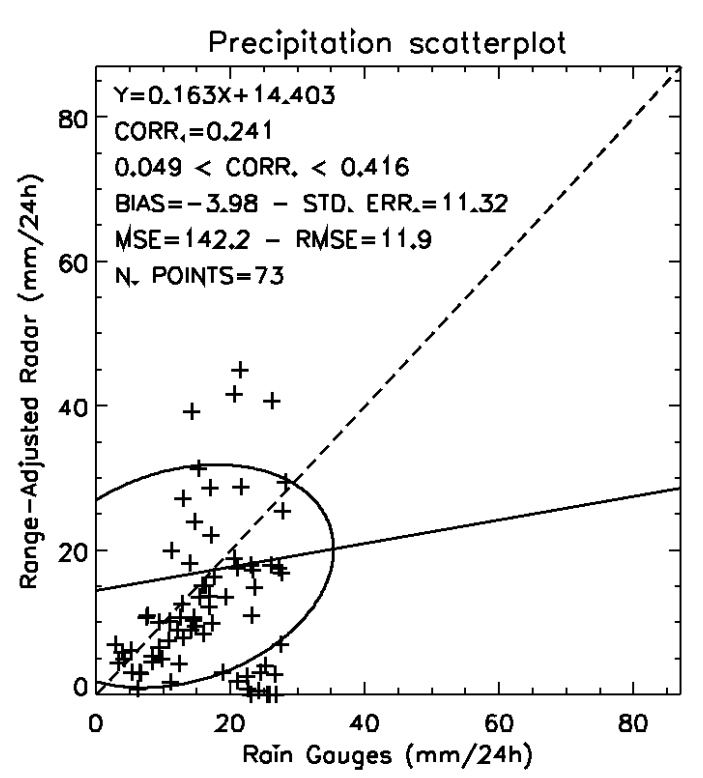

(b) RG gridded field vs. range-adjusted GR field

Fig. 3. Precipitation scatterplots of the 24 -h rain gauge gridded field against the 24-h radar field over 73 grid points where both data were available. Correlation (CORR.), and its confidence interval (Fisher, 1925), bias (BIAS), standard error (STD. ERR.), mean square error (MSE), root mean square error (RMSE) are indicated. A linear regression fit (solid line) and a 95\% confidence ellipse are also shown. 


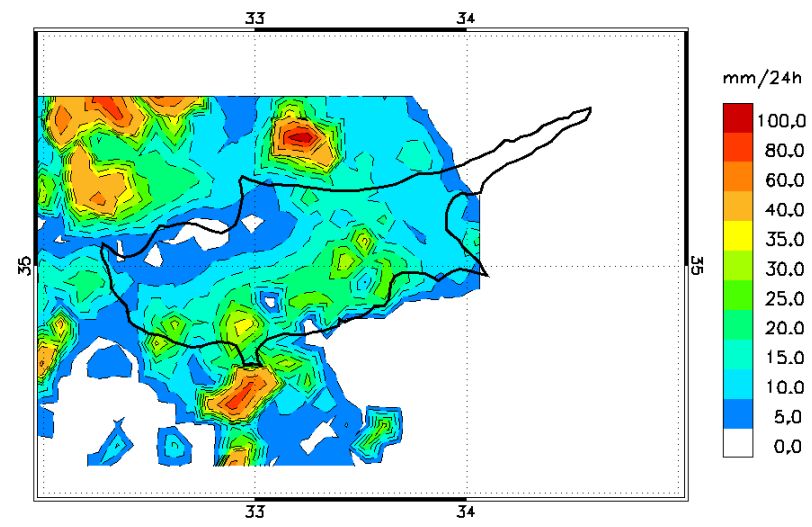

Fig. 4. The 24-h observational precipitation contours (mm) from 06:00 UTC 5 March to 06:00 UTC 6 March 2003, obtained according to Eq. (3).

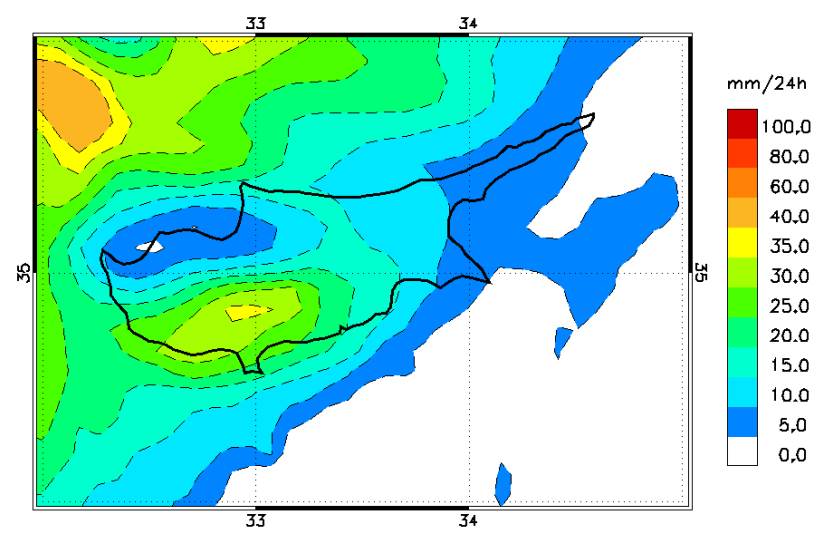

Fig. 5. The 24-h BOLAM forecast field contours $(\mathrm{mm})$ from 06:00 UTC 5 March to 06:00 UTC 6 March 2003.

observed precipitation field, which was used as verification grid.

Visual comparison between forecast and observational analysis (Fig. 6) displays a fair agreement, although the correlation is still low. Almost all the pairs outside the 95\% confidence ellipse (see Fig. 6) are related to the higher values of the observed precipitation, mainly due to GR data; RG did not exceed $39 \mathrm{~mm}(24 \mathrm{~h})^{-1}$.

\section{Methodology}

In order to give a quantitative assessment of the model's skill in predicting the event, non-parametric skill scores, such as ETS, BIA, HK, ORSS, POD and FAR, were initially used. These scores (for details see Hanssen and Kuipers, 1965; Schaefer, 1990; Wilks, 1995; Stephenson, 2000), which are tallied upon $2 \times 2$ contingency tables, summarize in a categorical way possible combinations of forecast and observed events above or below selected precipitation thresholds. The

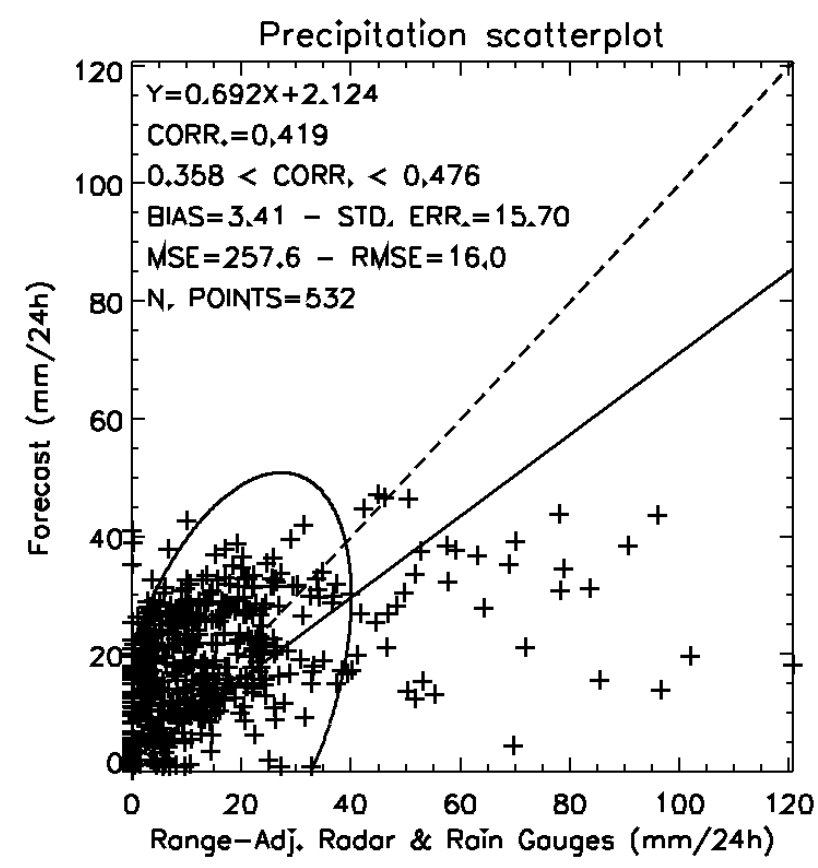

Fig. 6. As in Fig. 3, but for the 24-h forecast field against the 24-h observational field over 532 grid points.

set of thresholds, depending on the event magnitude, does not include values greater than $40 \mathrm{~mm}(24 \mathrm{~h})^{-1}$.

However, single case-study verification only by means of these scores may lead to unstable results, due to the limits of the statistical sample. Thus, the CRA analysis (Ebert and McBride, 2000), allowing to assess the spatial structure of the forecast error, was also performed. This object-oriented method is simply based on pattern matching of two contiguous areas, defined as the observed and forecast precipitation areas, delimited by a chosen isohyet, which was set to $0.5 \mathrm{~mm}(24 \mathrm{~h})^{-1}$. In a complex rain field (such as the one in question), it is preferable to match the rainfall patterns instead of the precipitation maxima, thus the correlation maximization was employed as the CRA pattern-matching criterion. The CRA analysis is performed by shifting the forecast precipitation field both latitudinally and longitudinally.

In order to eliminate matches that are not statistically significant, a threshold minimum correlation value between shifted forecast and observations was imposed. This value depends on the effective number of independent samples used in the comparison, which is a function of the number of grid points where the analysis is performed (this can change from shift to shift) and the autocorrelation of both the observed and forecast fields. The F test, with a $95 \%$ confidence level, was then applied to assess the statistical significance of each match (see Panofsky and Brier, 1958; Xie and Arkin, 1995). 
Table 1. Skill score results of comparison between the BOLAM forecast and the rain gauge analysis for the 5 March 2003 event. Skill score values less or equal zero are not indicated.

\begin{tabular}{ccccccc}
\hline $\begin{array}{c}\text { Threshold } \\
\mathrm{mm}(24 \mathrm{~h})^{-1}\end{array}$ & BIA & ETS & POD & FAR & HK & ORSS \\
\hline 5.0 & 1.014 & 0.739 & 1.000 & 0.014 & 0.750 & - \\
10.0 & 1.070 & 0.479 & 0.965 & 0.098 & 0.590 & 0.957 \\
15.0 & 1.275 & 0.347 & 0.925 & 0.274 & 0.501 & 0.887 \\
20.0 & 1.370 & 0.133 & 0.667 & 0.513 & 0.254 & 0.479 \\
40.0 & - & - & - & - & - & - \\
\hline
\end{tabular}

Table 2. Skill score results of comparison between the BOLAM forecast and the observational field (rain gauges + radar) for the 5 March 2003 event.

\begin{tabular}{ccccccc}
\hline $\begin{array}{c}\text { Threshold } \\
\mathrm{mm}(24 \mathrm{~h})^{-1}\end{array}$ & BIA & ETS & POD & FAR & HK & ORSS \\
\hline 5.0 & 1.284 & 0.159 & 0.947 & 0.262 & 0.230 & 0.753 \\
10.0 & 1.517 & 0.182 & 0.922 & 0.392 & 0.306 & 0.762 \\
15.0 & 1.732 & 0.160 & 0.799 & 0.539 & 0.326 & 0.631 \\
20.0 & 1.723 & 0.131 & 0.638 & 0.629 & 0.288 & 0.531 \\
40.0 & 0.243 & 0.136 & 0.162 & 0.333 & 0.156 & 0.939 \\
\hline
\end{tabular}

\section{Results and conclusions}

It is now possible to match the obtained results into a coherent picture. Model skill scores calculated with respect to the RG gridded analysis (i.e., only over the island of Cyprus) are fairly good (Table 1), whereas when including the GR range-adjusted data (i.e., also over the sea) quite poor scores are obtained (Table 2).

There are many reasons which could account for such differences in skill scores. For example, when comparing two fields, obtaining good skill scores when the area is large (and more than one type of observation is included) is very unlikely; whereas, it is easy to obtain good skill scores on smaller arear (in particular when only one type of observation is included). This result could also indicate a major impact of the observational error (e.g., GR range-effect, RG representativity error) on the skill scores when using the combined observational data set. Although we do not present the results in this paper, it is worth mentioning that the worst skill scores were obtained by using in the observational composite (i.e., RG + GR) original GR data (rather than those obtained by using range-adjusted GR data). This result also appears in Fig. 3a, where the precipitation scatterplot of the original GR data versus the RG data exibits the worst indicators (correlation and MSE). This confirms that radar range adjustment is a key procedure for having reliable GR data. Besides, BOLAM tends to overforecast precipitation almost everywhere (see the BIA score in Tables 1 and 2 and the 95\% confidence ellipse in Fig. 6), especially over the sea. This could indi-

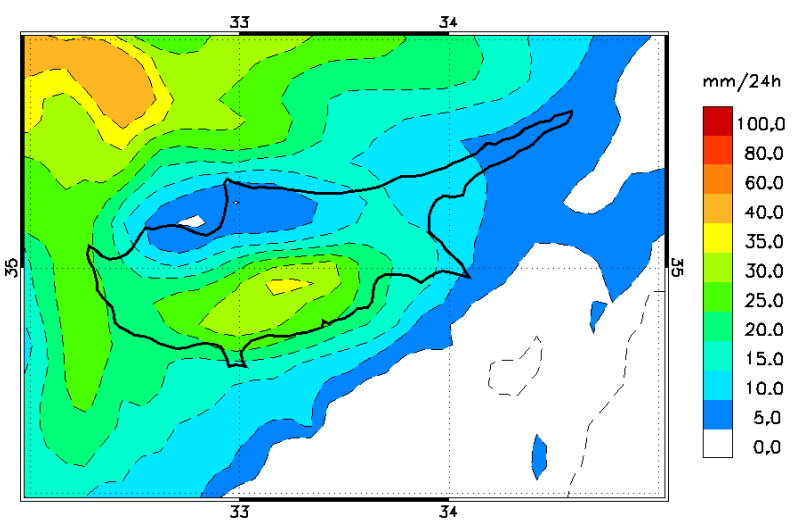

(a) Forecast shifted $0.27^{\circ} \mathrm{E}$ and $0.09^{\circ} \mathrm{N}$ with respect to the Barnes rain gauge-based analysis

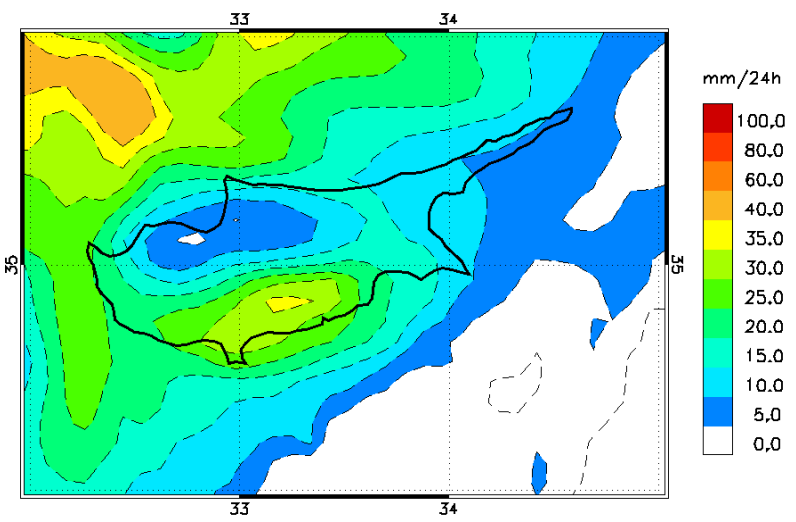

(b) Forecast shifted $0.27^{\circ} \mathrm{E}$ and $0^{\circ} \mathrm{N}$ with respect to the RGGR composite analysis

Fig. 7. The 24-h BOLAM forecast field contours (mm) from 06:00 UTC 5 March to 06:00 UTC 6 March 2003, as result of the CRA analysis.

cate that forecast rainfall qualitatively changes from land to sea, but this may be proven only on the basis of a long-term statistical verification of BOLAM over Cyprus.

Furthermore, model skill scores may be penalized by the forecast oversmoothness ${ }^{1}$. In fact, many details that are visible in the analysis over sea are not present in the forecast field (see in Fig. 4 the rainfall peaks located south, southwest and north-east of the island, respectively, and compare with Fig. 5). However, such discrepancies (and their effect on skill scores) cannot be unequivocally attributed to forecast error, since the observational error (namely, the radar range-effect) may sensibly affect the estimation of maximum rainfall peaks.

For what concerns the CRA results, when using the RG gridded analysis for verification, a better agreement (using

\footnotetext{
${ }^{1}$ Numerical models oversmooth precipitation fields with respect to reality (Chéruy et al., 2004).
} 
correlation maximization) between observations and forecast fields is obtained by shifting the forecast field $0.27^{\circ} \mathrm{E}$ and $0.09^{\circ} \mathrm{N}$ (see Fig. 7a against Fig. 2 and Tartaglione et al., 2005). This result is quite similar to the one obtained by using the RG-GR composite for verification. In fact, CRA results show that shifting $0.27^{\circ}$ eastward, the BOLAM forecast gives a better match with the observational analysis (correlation increases from 0.36 to 0.43 ).

An a priori estimate of the impact of the possible observational error on these results is not possible, thus we propose a subjective check of the analysis (Fig. 4) against the original and shifted forecast (Figs. 5 and 7b). The idea is to identify the qualitative features of the analysis illustrated in Fig. 4 which are less prone to possible observational error, and to check them against both the non-shifted and shifted model fields. For instance, it is quite improbable that radar errors introduce a macroscopic pattern shifting.

The gross structure of the rainy area on the island's southwestern side and of the non-rainy area on the northwestern side is better matched by the shifted forecast than by the original one. Over the sea, the check is difficult since a few observed peaks, probably due to convective activity, are absent in the BOLAM forecast (the hydrostatic model did not forecast them at all!). However, the position of the main rainy area, located to the northwest of the island, is better centred in the shifted forecast than in the original one.

The proposed results are a starting point for future works. The effect of forecast oversmoothness on the skill scores could be tested. Besides, the effect of different possible range adjustment techniques on the observational analysis could be also studied, in order to estimate and reduce the observational error and to assess the physical reliability of the CRA results. Overall, the results point out how much caution is needed when dealing with rainfall observations as the "ground truth". When satellite, rain gauge and radar data are simultaneously available over a large area, a reliable data base suitable for precipitation forecast verification can be built. Otherwise, the model verification task can be quite difficult, since it cannot be easy to distinguish the effect on the verification results of the forecast error from the one related to the observational error. Moreover, even a rough estimate of the observational error magnitude cannot be available. In such a condition, exemplified in the here presented case study, both analysis and forecast should be regarded as an error-affected representation of an unknown reality.

Acknowledgements. This research was supported by the EU Framework 5th Programme - Part A: Environment and Sustainable Development Contract EVK2-2002-CT-00155 (VOLTAIRE project). Authors are grateful to ECMWF for initial and boundary conditions for BOLAM. Comments and suggestions received during VOLTAIRE workshops were very useful.

\section{References}

Accadia, C., Mariani, S., Casaioli, M., Lavagnini, A., and Speranza, A.: Sensitivity of precipitation forecast skill scores to bilinear interpolation and a simple nearest-neighbor average method on high-resolution verification grids, Wea. Forecast., 18, 918-932, 2003.

Baldwin, M.: Quantitative Precipitation Forecast Verification Documentation, Tech. rep., NCEP/EMC, www.emc.ncep.noaa.gov/ mmb/ylin/pcpverif/scores/docs/mbdoc/pptmethod.html, 2000.

Barnes, S. L.: A technique for maximizing details in numerical weather map analysis, J. Appl. Meteorol., 3, 396-409, 1964.

Barnes, S. L.: Mesoscale objective analysis using weighted timeseries observations, NOAA, National Severe Storm Laboratory, Norman, OK 73069, tech. memo. ERL NSSL-62, 1973.

Buzzi, A. and Foschini, L.: Mesoscale meteorological features associated with heavy precipitation in the southern Alpine region, Meteorol. Atmos. Phys., 72, 131-146, 2000.

Chéruy, F., Speranza, A., Sutera, A., and Tartaglione, N.: Surface winds in the Euro-Mediterranean area: The real resolution of numerical grids, Ann. Geophys., 22, 4043-4048, 2004.

Ebert, E. E. and McBride, J. L.: Verification of precipitation in weather systems: determination of systematic errors, J. Hydrol., 239, 179-202, 2000.

Fisher, R. A.: Statistical Methods for Research Workers, Oliver and Boyd, Edinburgh (UK), 1925.

Gabella, M., Joss, J., Perona, G., and Michaelides, S.: Range adjustment for Ground based Radar, derived with the space-borne TRMM Precipitation Radar, IEEE Transactions on Geoscience and Remote Sensing, 44, 1, 126-133, doi:10.1109/TGRS.2005.858436, 2006.

Hanssen, A. W. and Kuipers, W. J. A.: On the relationship between the frequency of rain and various meteorological parameters, Meded. Verh., 81, 2-15, 1965.

Koch, S. E., desJardins, M., and Kocin, P. J.: An interactive Barnes objective map analysis scheme for use with satellite and conventional data, J. Clim. Appl. Meteor., 22, 1487-1503, 1983.

Malguzzi, P. and Tartaglione, N.: An economical second order advection scheme for numerical weather prediction, Quart. J. Roy. Meteorol. Soc., 125, 2291-2303, 1999.

Panofsky, H. A. and Brier, G. W.: Some applications of statistics to meteorology, Pennsylvania State University, University Park, 1958.

Schaefer, J. T.: The critical success index as an indicator of warning skill, Wea. Forecast., 5, 570-575, 1990.

Stephenson, D. B.: Use of the "Odds Ratio" for diagnosing forecast skill, Wea. Forecast., 15, 221-232, 2000.

Tartaglione, N., Mariani, S., Accadia, C., Speranza, A., and Casaioli, M.: Comparison of raingauge observations with modeled precipitation over Cyprus using contiguous rain area analysis, Atmos. Chem. Phys., 5, 2147-2154, 2005.

Wilks, D. S.: Statistical methods in the atmospheric sciences: An introduction, Academic Press, San Diego (USA), 1995.

Xie, P. and Arkin, P. A.: An intercomparison of gauge observations and satellite estimates of monthly precipitation, J. Appl. Meteorol., 34, 1143-1160, 1995. 